\title{
EFECTOS DE LA UNIÓN \\ CAMBIARIA SOBRE LOS \\ DIFERENCIALES DE RENTABILIDIDAD DE LA UNIÓN EUROPEA-15
}

\author{
Marta Gómez-Puig* \\ Universitat de Barcelona \\ Abril 2007
}

\begin{abstract}
Resumen
El efecto inmediato de la instauración de la Unión Monetaria Europea (UME), fue un aumento en los diferenciales de rentabilidad ajustados por el riesgo de cambio de la deuda pública frente a Alemania para aquellos países que se integraron en la moneda única. Contrariamente, para aquellos países que optaron por permanecer fura de la misma de forma inmediata se produce una disminución en los citados diferenciales de rentabilidad ajustados. El objetivo del presente trabajo es analizar cuáles pueden ser los motives que subyacen a este fenómeno. La evidencia empírica sugiere que la relevancia de la liquidez y de las economías de escala ligadas al tamaño del mercado han aumentado con la UME en todos los países de la EU-15.
\end{abstract}

Códigos de Clasificación JEL: E44, F36, G15.

Palabras clave: Integración Monetaria, Mercados de deuda pública, riesgo de crédito, liquidez del mercado y riesgo internacional.

\footnotetext{
Departament de Teoria Econòmica. Universitat de Barcelona. Diagonal, 690. Barcelona 08034. Spain. T: 34-934.021.935. Fax: 34-
} 934.039.082. E-mail: marta.gomezpuig@ub.edu. 


\section{Introducción}

Dos de los principales factores determinantes de los diferenciales de rentabilidad en los países participantes de la Unión Monetaria Europea (UME) fueron eliminados con la introducción de la moneda única en Enero de 1999 y la eliminación (o reducción hasta niveles insignificantes) de las diferencias en la fiscalidad sobre los intereses durante la década de los noventa. Este hecho implicó un aumento tanto en el grado de sustitución entre emisiones como en la relevancia del riesgo de crédito y de las diferencias de liquidez en los diferenciales de rentabilidad. No obstante, la segmentación distó mucho de desaparecer totalmente, máxime si, como hacemos en este trabajo, únicamente analizamos el efecto inmediato (durante los tres años posteriores) de la introducción de la moneda única. La persistencia de los diferenciales representa un claro ejemplo de la misma. En este sentido, a partir de nuestra muestra que está compuesta de datos diarios desde enero de 1996 hasta diciembre de 2001 (abarca los tres años anteriores y posteriores a la introducción del Euro), podemos extraer las conclusiones que se plasman en las figuras $1 \mathrm{a}$ 3 relativas a los países no participantes en la UME y que se suman a las ya comentadas en Gómez-Puig (2006a y 2006b) con relación a los países que se integraron en el euro. En concreto, la figura 1 muestra que para los países no participantes en el Euro, también los diferenciales de rentabilidad de la deuda pública a 10 años frente a Alemania experimentaron una importante convergencia durante los tres primeros años de moneda única. Adicionalmente, la disminución del diferencial de rentabilidad durante ese período resulta más que el doble del experimentado por los países de la Euro-zona (32,99 puntos básicos en promedio). En concreto, el diferencial promedio de rentabilidad de los países no participantes en el Euro experimentó una reducción de 73,06 puntos básicos, desde 100,81 en el período pre-UME considerado, hasta 27,75 en los tres primeros años de Unión Monetaria (véase la tabla 1). Por su parte, la evolución de los diferenciales de tipo swap a 10 
años frente a Alemania también muestra un comportamiento claramente divergente en los dos grupos de países. Así, para el caso de los países que permanecieron fuera de la moneda única, la diferencia en su evolución con respecto a los de los países de la zona euro (véase Gómez-Puig 2006a y 2006b) que ya era importante antes de la moneda única se vio acentuada con la entrada en vigor de la misma y la importante convergencia hasta valores próximos a cero experimentada por los diferenciales de tipos swap en los países de la UME (véase figura 2). De ese modo, la evolución temporal de la variable que como en nuestros trabajos anteriores utilizamos como variable dependiente: "el diferencial de rentabilidad ajustado por el riesgo de cambio" (considerando el diferencial entre tipos swap una buena aproximación del citado riesgo) presenta, a partir de enero de 1999, una evolución completamente diferente para los países pertenecientes al Euro y no pertenecientes al mismo (véase figura 3). Ciertamente, la tabla 1 muestra una sustancial convergencia de los diferenciales brutos de rentabilidad a 10 años frente a Alemania durante el período mencionado. No obstante, la convergencia únicamente implicó una reducción del coste relativo del endeudamiento para el caso de aquellos países que con anterioridad presentaban superiores diferenciales, inferior rating, y mayor riesgo de cambio. Contrariamente, los países que menos se beneficiaron de la eliminación del riesgo de cambio experimentaron un aumento en el coste relativo de su endeudamiento. Por su parte, los tres países de la Europa de los quince que no se incorporaron a la moneda, ajenos a la creciente competencia entre los mercados de deuda pública europeos parecen haber disfrutado de una menor valoración de su prima de riesgo y de una mayor valoración de sus particulares idiosincrasias por parte de los agentes del mercado. Este hecho provocó un trasvase de fondos hacia estos mercados de la mano de aquellos inversores que deseaban continuar disminuyendo el riesgo total de su cartera de inversión a través de la diversificación. Así, en promedio, los tres países que no se integraron en el Euro, 
experimentaron una reducción en sus diferenciales brutos de rentabilidad que más que dobla la registrada por parte de los países de la zona-Euro (véase tabla 1).

Nuestra muestra incluye todos los países de la EU-15 (i.e. tanto aquellos que se integraron en la moneda única como aquellos que prefirieron permanecer ajenos a la misma), con la excepción de Luxemburgo y Grecia. Por consiguiente, con el objetivo de poder trabajar con series homogéneas a lo largo de los seis años que componen la muestra tanto para los países no participantes en el euro como para los participantes (en ellos el riesgo de cambio fue eliminado en enero de 1999), vamos a seguir el modelo de Favero, Giavazzi y Spaventa (1997) y corregiremos los diferenciales de rentabilidad aproximando el riesgo de cambio al diferencial entre los tipo swap a 10 en la moneda de denominación de cada uno de los bonos y el diferencial de tipos swap a 10 años en marcos alemanes (para una explicación y detalle más preciso de este ajuste, véase Gómez-Puig 2006a 2006b). Una vez los diferenciales han sido corregidos por el riesgo de cambio, durante los tres primeros años de Unión Monetaria, observamos resultados que merecen el calificativo de sorprendentes (véase tabla 1). Los países no participantes en el euro experimentaron un descenso promedio de 14,20 puntos básicos en sus diferenciales de rentabilidad ajustados, mientras que los países que se adhirieron a la moneda única registraron un incremento promedio de 11,98 puntos básicos. Identificar los posibles factores que subyacen a estos resultados es el principal objetivo de nuestro análisis, i.e. evaluar qué factores contribuyeron al mencionado efecto inmediato de la introducción de la moneda única en la evolución de los diferenciales de rentabilidad en todos los países que componen la EU-15. El resto del trabajo se organiza de la siguiente manera. La Sección 2 subraya algunas consideraciones acerca de los factores susceptibles de incidir sobre los diferenciales de rentabilidad ajustados. La Sección 3 describe los datos. La sección 4 explica el método de estimación y resume los resultados obtenidos. Finalmente, en la sección 5 se extraen las principales conclusiones 


\section{Factores explicativos de los diferenciales de rentabilidad ajustados.}

$\mathrm{Al}$ igual que en nuestros trabajos anteriores, la variable que utilizaremos como variable dependiente, $A \operatorname{SPRE} A D_{i t}$ (la diferencia entre el diferencial bruto de rentabilidad a 10 años y el diferencial entre los tipos swap a 10 años) básicamente recogerá aquellos factores diferentes del riesgo de cambio susceptibles de incidir en el diferencial de rentabilidad. En particular, en este trabajo vamos a evaluar la importancia relativa de los factores de riesgo doméstico (principalmente riesgo de crédito y diferencias en la liquidez del mercado de los diferentes países frente a Alemania) y de los factores de riesgo internacional.

Así, en los países de la zona-euro, un primer punto importante a valorar es si la UME ha contribuido a incrementar el riesgo de crédito al eliminar a los gobiernos la salida de emergencia que suponía la posibilidad de monetizar la deuda y al prohibir tanto al BCE como a la UE ejercer de salvaguarda de los gobiernos en apuros financieros. O si, contrariamente, el umbral máximo que la UME ha impuesto a los gobiernos tanto para su nivel de endeudamiento como para su nivel de déficit público junto con la posibilidad de que los agentes otorguen una baja credibilidad a la cláusula de no salvaguarda, se ha traducido en una disminución del riesgo de crédito percibido. Nuestro propósito es comparar los resultados obtenidos para los países de la zona euro con aquellos países integrantes de la EU-15 que en enero de 1999 no se adhirieron a la moneda única. En segundo lugar, la eliminación del riesgo de cambio derribó una importante barrera que anteriormente había estado protegiendo a los diferentes mercados domésticos. En consecuencia, en este nuevo contexto de superior competitividad entre los mercados de deuda pública de la zona Euro, su éxito podría verse limitado por su grado de liquidez y el tamaño de sus mercados (véase Gómez-Puig 2006a). Contrariamente, ajenos a la creciente competitividad en la zona euro, los países que mantuvieron en 1999 su propia divisa, podrían haberse beneficiado de un incremento de la valoración otorgada por los mercados a su renovada singularidad e idiosincrasias, atrayendo inversiones hacia sus mercados. En 
particular, el mercado británico, el cuarto en tamaño de la EU-15, podría seguramente ser el que en mayor medida habría capitalizado esta ventaja comparativa. Este es el segundo punto que analizaremos en este trabajo. En este sentido, existe literatura que apoya la importancia del tamaño del mercado en el éxito de un mercado de deuda pública. Martin y Rey (2004) muestran que, en general, el tamaño importa en los mercados de activos, de tal manera que cuánto mayor sea el tamaño del mismo, mayores serán los beneficios asociados a precios superiores. McCauley y Remolona (2000) señalan que si existen costes fijos importantes asociados a la obtención de información acerca de la evolución futura de los tipos de interés, el tamaño de todo el mercado es relevante. Por otro lado, Eonomides y Siow (1988) puntualizan que existe un trade-off entre liquidez y tamaño del mercado. De ese modo, si el tamaño influye en la liquidez, "ex-ante" los inversores preferirán invertir en mercados grandes y líquidos antes que en mercados pequeños e ilíquidos. Por consiguiente, la liquidez mostrará una naturaleza "retro-alimentadora". Finalmente, el análisis en este trabajo también se fundamentará en la reciente evidencia empírica acerca de los factores que determinan la evolución de los diferenciales de rentabilidad en los mercados emergentes. Esta evidencia sugiere que los diferenciales son especialmente sensibles a los factores de riesgo internacional (véase Codogno, Favero y Missale, 2003).

\section{Datos}

Tal como ha sido definida, la variable dependiente es $A S P R E A D_{i t}$, i.e. la diferencia entre el diferencial de bruto de rentabilidad de las emisiones soberanas a 10 años frente a Alemania y el diferencial entre los tipos swap a 10 años del país en cuestión y de Alemania. Las rentabilidades y los tipos swap han sido obtenidos de Datastream y corresponden a la emisión benchmark en cada momento del tiempo. El ratio Deuda Pública sobre PIB de cada país en relación a Alemania, será utilizado como proxy para medir el riesgo de crédito. La variable recibe la denominación de $L N D E B T G D P_{i t}$ El saldo total de de deuda pública de cada país ha sido obtenido del Banco Internacional de Pagos, mientras que los datos 
referentes al PIB han sido obtenidos de Eurostat (en ambos casos, estos datos se publican con una frecuencia trimestral, por consiguiente hemos asumido una tasa de crecimiento diaria constante en ambos casos para así extrapolar el resto de los datos). Por otro lado, hemos utilizado dos proxies para capturar las diferencias de liquidez: Los diferenciales Comprador/Vendedor, BIDASKDIF $F_{i t}$ y el diferencial entre la rentabilidad de la emisión benchmark en un momento determinado y la que fue benchmark justo en el momento anterior. Es decir el diferencial on-the-run/off-the-run, ONOFFDIF ${ }_{i r}$ En ambos casos, hemos creado series diarias a partir de los datos que proporciona Bloomberg. Finalmente, el diferencial entre el tipo de interés swap a 10 años y la rentabilidad de los bonos corporativos a 10 años con calificación AAA por parte de la agencia Moody (USSPRE $A D_{i}$ ), que ha sido calculado a partir de datos diarios obtenidos de Datastream, es la proxy que utilizaremos para capturar el riesgo internacional. Todas las variables incluidas en nuestras estimaciones que capturan diferentes tipos de riesgo doméstico están en términos relativos frente a Alemania.

\section{Modelos estimados y resultados.}

Presentaremos los resultados correspondientes a un mismo modelo que ha sido estimado para los dos grupos de países (participantes y no participantes en el euro) con el fin de poder comparar los resultados. Esta especificación es precisamente la que presentó el máximo poder explicativo en nuestros trabajos anteriores (Gómez-Puig, 2006a y 2006b). Se trata de una estimación estática con datos de panel que incluye las variables mencionadas en la sección 3 (por consiguiente, las variables independientes capturan tanto eventuales elementos de riesgo doméstico como de riesgo internacional). Debe señalarse que hemos formulado una especificación cuadrática en el caso de las variables que capturan diferencias en la liquidez de los mercados con el fin de evaluar si la liquidez presenta un comportamiento no-lineal. Por su parte la variable que recoge el riesgo internacional 
aparece en las regresiones tanto en forma lineal como interactuando con el resto de variables domésticas. De ese modo, el vector de variables de riesgo doméstico ( $\left.D R V_{i t}\right)$ será: $D R V_{i t}=\left(L_{N D E B T G D P}{ }_{i p} B I D A S K D I F_{i p} B I D A S K D I F 2_{i p}\right.$ ONOFFDIF $\left._{i p} \mathrm{ONOFFDIF}_{i t}\right)$

Mientras que el vector de variables de riesgo internacional (IRV $\left.V_{i}\right)$ será:

$I R V_{i t}=U S S P R E A D_{i t}$

Adicionalmente, en el modelo hemos incluido variables dummy mensuales y para cada uno de los países que integran los dos paneles, así como una variable dummy (DPRE) que toma el valor 1 en el periodo pre-EMU (y 0 , en caso contrario). De ese modo, los coeficientes de las interacciones entre esta variable dummy y el resto de las variables son calculados. Nuestra especificación es:

$y_{i t}=\alpha_{i}+\beta X_{i t}+\gamma$ DPRE $_{i t}+\delta M O N T H L Y D U M M I E S_{t}+\lambda$ COUNTRYDUMMIE $_{i}+\varepsilon_{i t}$

donde el vector de variables independientes es:

$X_{i t}=\left(I R V_{i s} D R V_{i s} D R V_{i t}^{*} I R V_{i t}\right)$

Adicionalmente : $\beta=\beta_{1}+\beta_{2} D P R E_{i t}$

Por consiguiente, el efecto marginal de una variable será : $\beta=\beta_{1}$ (en el período UME) y $\beta=\beta_{1}+\beta_{2}$ (en el periodo pre-EMU)

El método de estimación utilizado, Feasible Generalized Least Squares (FGLS), robusto a la posible existencia de autocorrelación o heterocedasticidad en los términos de error. La Tabla 2 presenta los resultados para los dos grupos de países: aquellos que participaron en el euro en 1999 y aquellos que decidieron permanecer ajenos a la moneda única. Los principales resultados acerca del impacto inmediato de la introducción de la moneda única para los países de la Euro-zona son los siguientes: Por un lado, se observa un aumento del efecto marginal de los términos lineales de las dos proxys utilizadas para medir las diferencias de liquidez en los mercados a partir del inicio de la Unión Monetaria. Por el otro, cuando es interaccionada con la variable de riesgo internacional (que experimenta un aumento en su efecto marginal con el euro), el comportamiento de la variable on-the- 
run/off-the-run es no-lineal, apoyando la posibilidad de una eventual naturaleza "retroalimentadora" para la liquidez. Con relación a la variable que captura el riesgo de crédito, resulta más significativa con el inicio de la UME. Por consiguiente, al menos para todos los países de la UME de forma global, estos resultados sugieren que la cláusula de no salvaguarda era creíble durante los tres primeros años tras la introducción del euro. En el caso de la estimación de panel para los países no pertenecientes al euro encontramos resultados similares con referencia a las variables relacionadas con las diferencias de liquidez en los mercados. Debe señalarse, no obstante que el diferencial on-the-run/ offthe-run no solo aumenta su impacto marginal con el euro, sino que también presenta un comportamiento no-lineal, acorde con una eventual naturaleza retro-alimentadora para la liquidez. Contrariamente, no solamente el impacto del riesgo de crédito no aumenta con la Unión Cambiaria, sino que su efecto marginal toma un valor negativo con la entrada del euro. Finalmente, en relación a la variable que captura el riesgo internacional, debe señalarse que el aumento registrado por su efecto marginal a partir de 1999 es superior que el experimentado por los países integrantes en la moneda única.

\section{Conclusiones}

Los resultados son plenamente consistentes con los obtenidos en nuestros anteriores estudios (Gómez-Puig 2006a y 2006b) y proporcionan evidencia empírica de que las economías de escala ligadas al tamaño parecen haber aumentado con la Unión Cambiaria en todos los países que conforman la Europa de los quince, al menos durante los tres primeros años de UME. De hecho, desde enero de 1999 el diferencial de interés ajustado de tipos de interés a 10 años frente a Alemania ha aumentado en todos los países de la zona euro y en aquel país que, si bien no forma parte del euro, mantiene una política cambiaria de estrecho vínculo de la evolución de la cotización de su moneda con la del euro y además posee un mercado de deuda pública muy pequeño (es el caso de Dinamarca). Por consiguiente, la eliminación de la barrera cambiaria parecería haber penalizado a los 
mercados más pequeños de deuda pública de la zona euro doblemente, ya que ahora deben pagar tanto una prima de riesgo como de liquidez superior a la de los mercados de superior tamaño. Por su parte, dos mercados: el Alemán y el Británico (el segundo y el cuarto en tamaño de la EU-15) podrían ser los mercados que en mayor medida habrían capitalizado los beneficios de esta nueva situación al atraer hacia los mismos un importante volumen de fondos. Además, en la zona Euro es de destacar que el impacto marginal de la variable que captura el riesgo de crédito aumenta en los tres años posteriores a 1999. Por consiguiente, la cláusula de no salvaguarda es creíble globalmente para todos los países de la zona euro durante el período analizado. Por su parte, los resultados también apoyan la naturaleza retro-alimentadora de la liquidez en los dos grupos de países, i.e. apoyan la idea de que las transacciones de los inversores fluyen desde los pequeños e ilíquidos mercados hacia los más grandes y más líquidos mercados. Finalmente, la relevancia de la variable que captura el riesgo internacional es superior en los países que no forman parte del euro. El hecho que estos países no compartan una politica monetaria común podría hacerlos más vulnerables a los factores de riesgo externo. Para concluir, la introducción de una moneda única y en el actual contexto de superior competitividad entre las emisiones soberanas de la zona euro, su éxito podría estar perfectamente ligado con el tamaño de su merado. Por su parte, los países ajenos al euro, que no se han visto expuestos a ese incremento en la competitividad, podrían haberse beneficiado del hecho de que los participantes del mercado hayan considerado que a partir de 1999 su prima de riesgo es más baja y los eventuales beneficios ligados a las inversiones en los mismos más elevados. 


\section{Referencias}

Codogno, L., C.Favero y A. Missale, 2003. EMU and Government Bond Spreads. Economic Policy n.18, pp.503-532.

Economides, N. y A.Siow, 1988. The Division of Markets is Limited by the Extent of Liquidity (Spatial Competition with Externalities). American Economic Review, Vol.78, n¹ pp 1719-1734.

Favero, C., F. Giavazzi y L. Spaventa, 1997. High Yields: The Spread on German Interest Rate. The Economic Journal, Vol.107, Issue 443.

Gómez-Puig, M., 2006a. Size Matters for Liquidity: Evidence from EMU sovereign yield spreads. Economics Letters, Vol.9, Issue 2; pp.156-162, February.

Gómez-Puig, M. 2006b. Monetary Integration and the Cost of Borrowing. Journal of International Money and Finance, en prensa.

Martin, P y H.Rey, 2004. Financial super-markets: size matters for asset trade. Journal of International Economics 64, pp. 335-361.

McCauley R. y E.Remolona, 2000. Size and Liquidity of Government Bond Markets. BIS Quarterly Review, November. 
Figura 1. Diferenciales de rentabilidad a 10 años frente a Alemania.

10 YEARS YIELD SPREADS OVER GERMANY

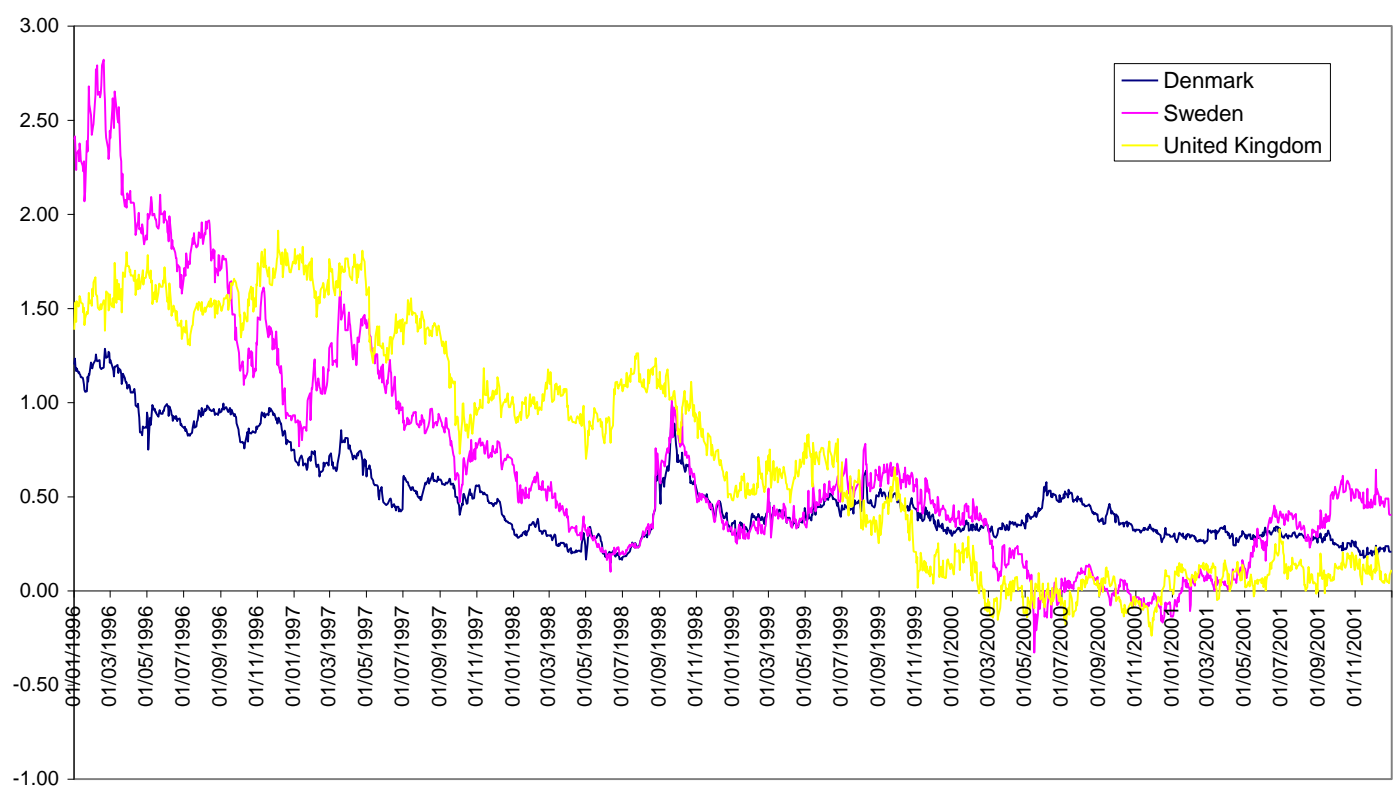

NOTA: Diferencial de Rentabilidad $=\left(\mathrm{I}_{\mathrm{i}}-\mathrm{I}_{\mathrm{DM}}\right)$, donde $\mathrm{I}_{\mathrm{i}}$ es la rentabilidad a 10 años de la deuda pública del país $i$ y $\mathrm{I}_{\mathrm{DM}}$ es la rentabilidad a 10 años de la deuda pública alemana. .

Fuente: Datastream 
Figura 2. Diferenciales de tipos swap a 10 años frente a Alemania.

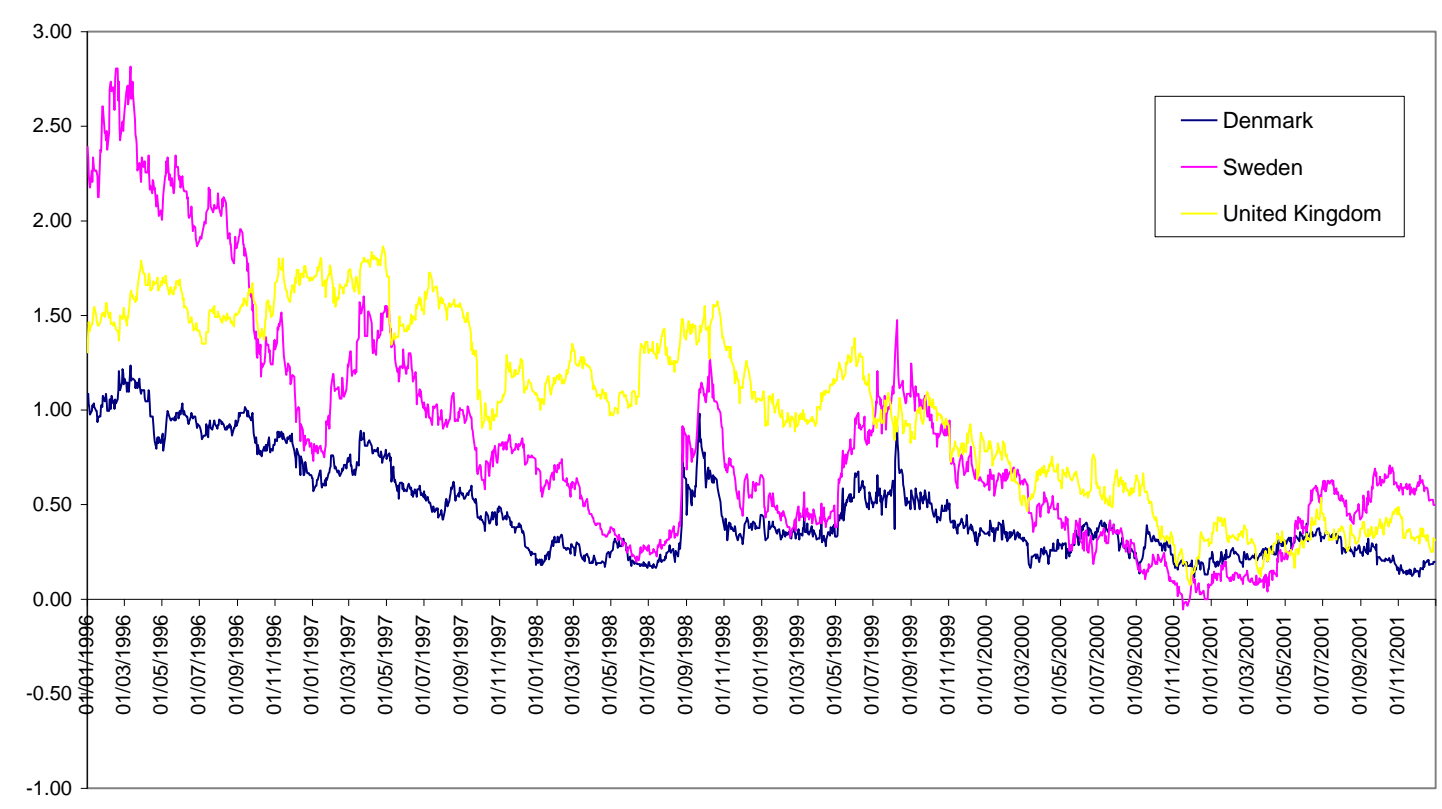

NOTA: Diferencial de tipos swap $=\left(I_{R} S_{i}-I R S_{D M}\right)$, donde $I_{R} S_{i}$ es el tipo de interés swap a 10 años de la moneda $i$ y IRS $_{\text {DM }}$ es el tipo de interés swap a 10 años de Alemania.

Fuente: Datastream 
Figura 3. Diferenciales de rentabilidad ajustados a 10 años frente a Alemania.

10 YERS ADJUSTED YIELD SPREADS OVER GERMANY

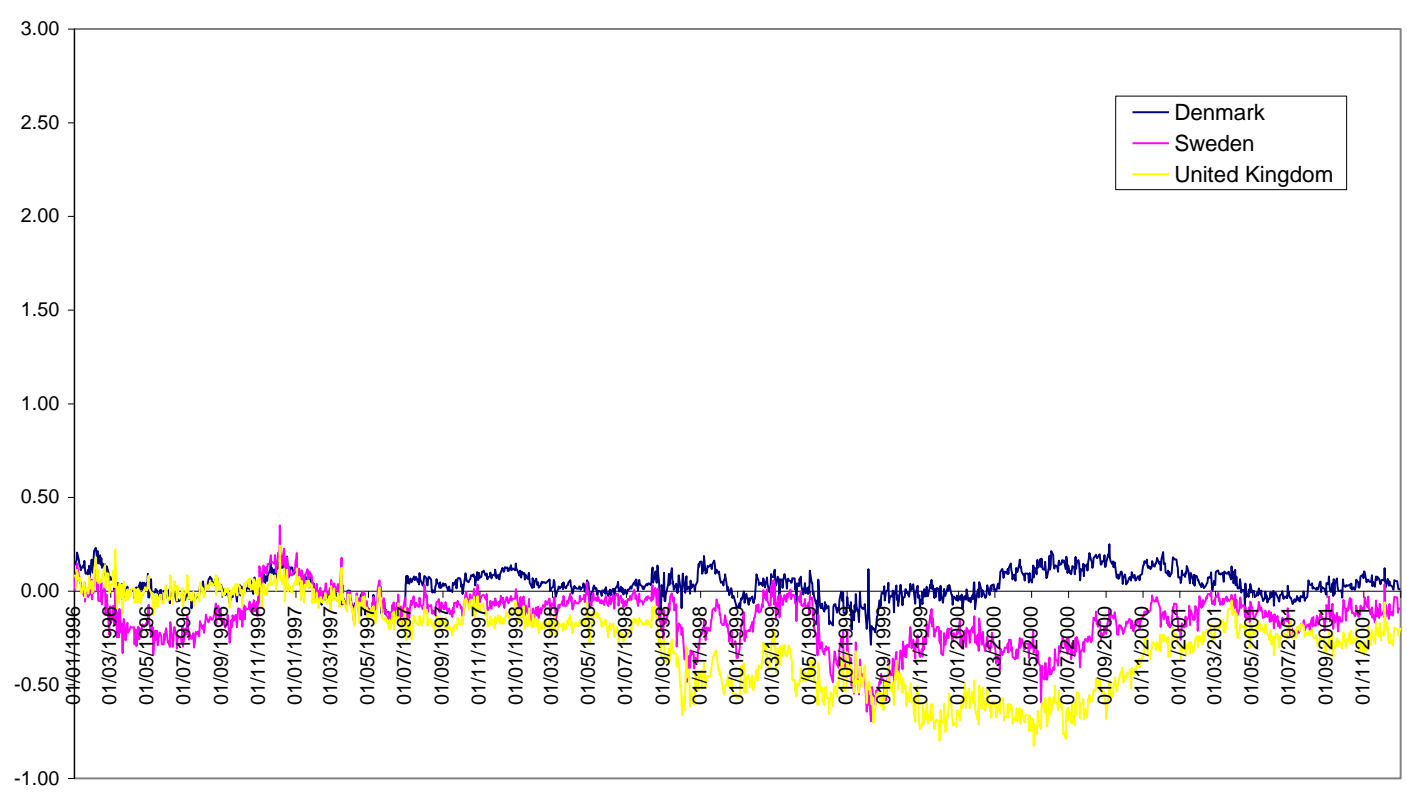

NOTA: Diferencial de Rentabilidad Ajustado $=$ Diferncial de Rentabiliad- Diferencial de tipos swpa $=\left(\mathrm{I}_{\mathrm{i}}-\mathrm{I}_{\mathrm{DM}}\right)-\left(\mathrm{IRS}_{\mathrm{i}}-\right.$ IRS $_{\text {DM) }}$. Fuente: Datastream. 
Tabla 1

\begin{tabular}{|c|c|c|c|c|c|c|c|c|}
\hline & \multicolumn{3}{|c|}{ PRE-UME (1996-1998) } & \multicolumn{3}{|c|}{ UME (1999-2001) } & \multicolumn{2}{|c|}{ Diferencias entre UME y pre-UME } \\
\hline & $\left(\mathrm{I}_{\mathrm{i}}-\mathrm{I}_{\mathrm{DM}}\right)$ & $\left(\right.$ IRS $\left._{\mathrm{i}}-\mathrm{IRS}_{\mathrm{DM}}\right)$ & ASPREAD $_{i}$ & $\left(\mathrm{I}_{\mathrm{i}}-\mathrm{I}_{\mathrm{DM}}\right)$ & $\left(\right.$ IRS $\left._{\mathrm{i}}-\mathrm{IRS}_{\mathrm{DM}}\right)$ & ASPREAD $_{\mathrm{i}}$ & $\left(\mathrm{l}_{\mathrm{i}}-\mathrm{I}_{\mathrm{DM}}\right)$ & ASPREAD $_{i}$ \\
\hline & (1) & (2) & $(3)=(1)-(2)$ & (4) & (5) & $(6)=(4)-(5)$ & (4)-(1) & (6)-(3) \\
\hline \multicolumn{9}{|l|}{ EURO } \\
\hline AT & 9.07 & -0.33 & 9.40 & 24.42 & -0.01 & 24.43 & 15.34 & 15.03 \\
\hline $\mathrm{BE}$ & 33.06 & 4.29 & 28.77 & 46.30 & -0.01 & 46.31 & 13.24 & 17.53 \\
\hline $\mathbf{F I}$ & 43.56 & 41.31 & 2.25 & 21.95 & -0.01 & 21.96 & -21.61 & 19.71 \\
\hline FR & 2.97 & -3.10 & 6.07 & 14.05 & 0.00 & 14.05 & 11.08 & 7.98 \\
\hline IE & 50.52 & 43.84 & 6.68 & 14.78 & 0.00 & 14.78 & -35.74 & 8.10 \\
\hline IT & 157.73 & 133.04 & 24.69 & 32.32 & 0.05 & 32.27 & -125.40 & 7.58 \\
\hline NL & -2.70 & -3.52 & 0.83 & 14.22 & -0.01 & 14.23 & 16.92 & 13.40 \\
\hline PT & 111.73 & 91.42 & 20.31 & 31.85 & 0.22 & 31.63 & -79.88 & 11.31 \\
\hline SP & 118.06 & 97.99 & 20.07 & 27.24 & 0.04 & 27.20 & -90.82 & 7.13 \\
\hline Promedio & 58.22 & 44.99 & 13.23 & 25.24 & 0.03 & 25.21 & -32.99 & 11.98 \\
\hline Desv.St. & 57.48 & 51.37 & 10.33 & 10.66 & 0.08 & 10.64 & 53.77 & 4.69 \\
\hline \multicolumn{9}{|l|}{ NO-EURO } \\
\hline DK & 64.01 & 61.09 & 2.92 & 35.83 & 32.61 & 3.23 & -28.18 & 0.31 \\
\hline sw & 108.48 & 117.15 & -8.67 & 28.14 & 49.01 & -20.87 & -80.33 & -12.20 \\
\hline UK & 129.95 & 142.05 & -12.10 & 19.27 & 62.07 & -42.80 & -110.68 & -30.70 \\
\hline Promedio & 100.81 & 106.77 & -5.95 & 27.75 & 47.90 & -20.15 & -73.06 & -14.20 \\
\hline Desv.St. & 33.63 & 41.46 & 7.87 & 8.29 & 14.76 & 23.02 & 41.73 & 15.60 \\
\hline
\end{tabular}

NOTA: AT: Austria, BE: Bélgica, FI: Finlandia, FR: Francia, IE: Irlanda, IT: Italia, NL: Holanda, PT: Portugal, SP: España, DK: Dinamarca, SW: Suecia, UK: Reino Unido. Fuente: Datastream. $\left(\mathrm{I}_{\mathrm{i}-} \mathrm{I}_{\mathrm{DM}}\right)=$ Diferencial a 10 años frente a Alemania. $\left(\mathrm{IRS}_{\mathrm{i}}-\mathrm{IRS}_{\mathrm{DM}}\right)=$ Diferencial a 10 años de los tipos swap frente a Alemania. ASPREAD $\mathrm{D}_{\mathrm{i}}=\left(\mathrm{I}_{\mathrm{i}}-\mathrm{I}_{\mathrm{DM}}\right)-\left(\mathrm{IRS}_{\mathrm{i}}-\right.$ IRS $\left._{\mathrm{DM}}\right)$ 
Tabla 2

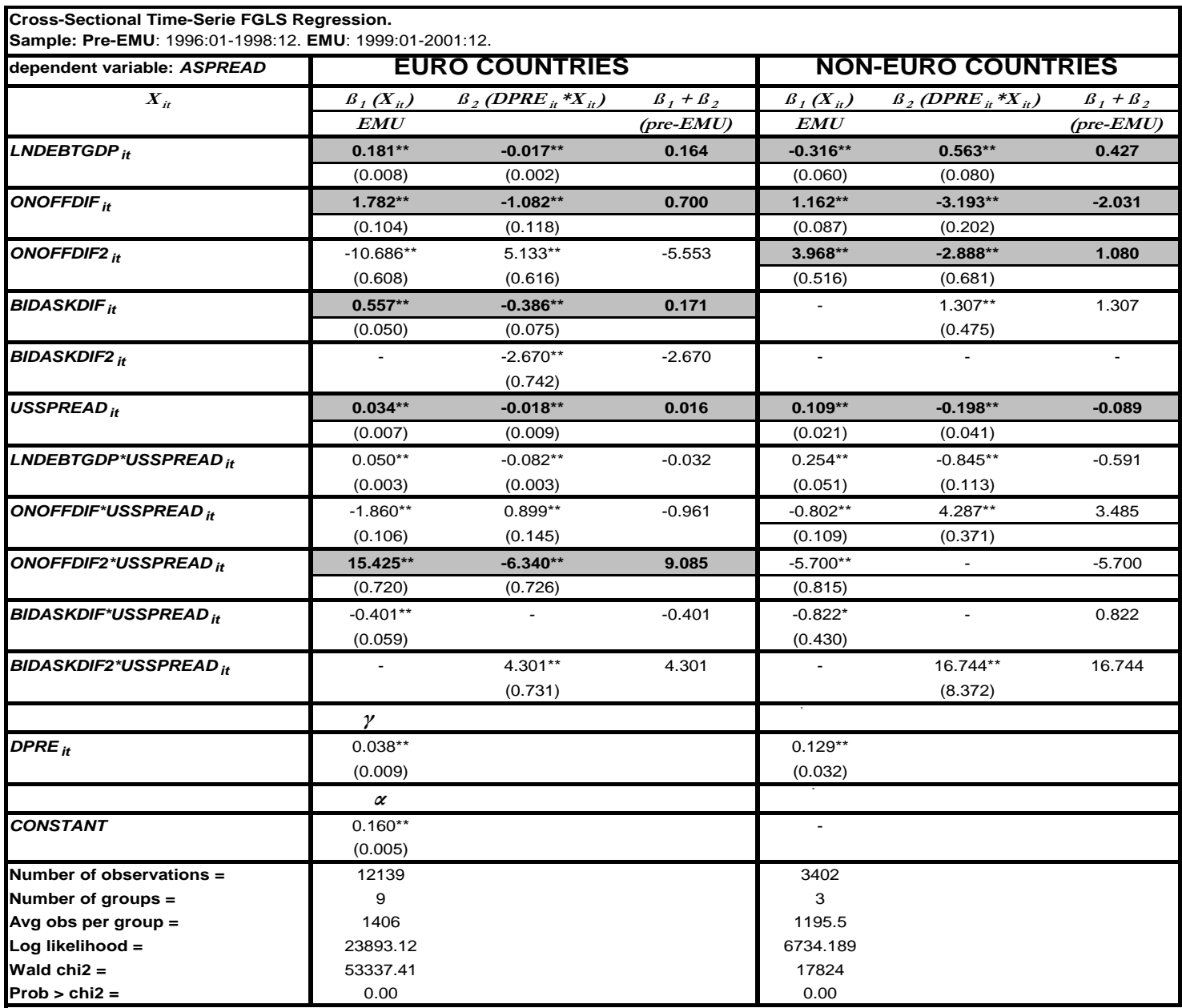

**Significant at 5 percent confidence level. *Significant at 10 percent confidence level.

Standard Errors within parentheses 